Editorial

\title{
Applied Sciences Special Issue: Ultrasonic Guided Waves
}

\author{
Clifford J. Lissenden
}

Department of Engineering Science and Mechanics, Pennsylvania State University, University Park, PA 16802, USA; lissenden@psu.edu

Received: 9 September 2019; Accepted: 10 September 2019; Published: 15 September 2019

The propagation of ultrasonic guided waves in solids is an important area of scientific inquiry due primarily to their practical applications for the nondestructive characterization of materials, such as nondestructive inspection, quality assurance testing, structural health monitoring, and for achieving material state awareness. This Special Issue of the journal covers all aspects of ultrasonic guided waves (e.g., phased array transducers, meta-materials to control wave propagation characteristics, scattering, attenuation, and signal processing techniques) from the perspective of modeling, simulation, laboratory experiments, or field testing. In order to fully utilize ultrasonic guided waves for these applications, it is necessary to have a firm grasp of their requisite characteristics, which include being multimodal, dispersive, and comprised of unique displacement profiles through the thickness of the waveguide.

The majority of the manuscripts in this Special Issue report on waveguides that are pipes and plates, but rods, rail, multi-strand wire, human bone, and half-spaces are also investigated. Hakoda et al. [1] provide experimental results on an aluminum plate, demonstrating how wave propagation can be manipulated by changing the boundary conditions. Specifically, surface-mounted resonators block the propagation of the S0 Lamb mode at low frequencies. Forbidding the propagation of the S0 Lamb mode turns out to be very different from forbidding the propagation of the A0 Lamb mode but can be explained in terms of the boundary conditions, which provide an alternative approach to meta-materials. The remaining 18 manuscripts pertain directly to the detection of defects within the waveguide in order to enable the assessment of the structural integrity.

While the purpose of pipes is to transport or circulate fluids, their hollow cylindrical geometry forms an efficient waveguide for longitudinal, torsional, and flexural ultrasonic waves. Torsional waves are often preferred for long-range defect detection because the wave energy does not leak into the fluids inside the pipe. Six manuscripts in the Special Issue investigate ultrasonic guided waves in pipes for the purpose of defect detection. Rose et al. [2] show that an array of fiber Bragg grating (FBG) sensors multiplexed along an optical fiber can receive torsional guided waves in a pipe, thus improving the range of piezoelectric and magnetostrictive actuators because reflections do not have to propagate back to the actuator. Pedram et al. [3] demonstrate the use of split-spectrum processing to improve the signal-to-noise ratio (SNR) of axisymmetric torsional waves in pipes and investigate its limitations. They show a $30 \mathrm{~dB}$ increase in SNR. Mahal et al. [4] first propose a method to detect defects in pipes from axisymmetric torsional waves having a low signal-to-noise ratio by leveraging the signals received from individual elements in the ring array transducer. Then, they [5] propose an adaptive leaky normalized least-mean-square filter to reduce the effect of unwanted flexural waves on the detection of axisymmetric waves. In a third paper [6], the same authors describe the exploitation of the power spectrum to distinguish torsional waves from flexural waves in pipes for more reliable defect detection. Taking a different approach, $\mathrm{Wu}$ and Lee [7] propose applying a multilayer perceptron neural network to detect leaks in pressurized natural gas pipelines, based upon acoustic emissions from the leaks. Accurate localization depends on the group velocity of the flexural waves generated during the acoustic emission events. 
The Lamb waves and shear-horizontal (SH) waves that propagate in traction-free homogeneous isotropic plates are often used for defect detection when access is limited or to cover a large area. Hakoda and Lissenden [8] demonstrate how the analysis of partial waves provides new perspectives on the propagation characteristics of elastodynamic guided waves in plates as well as other types of waveguides. Fan et al. [9] employ wavenumber filtering to remove Lamb modes other than the A0 mode to detect flat bottom holes in a plate. Wu et al. [10] propose a methodology for nondestructive inspection of cylindrical storage tanks' base plates whereby a transducer is scanned around the perimeter of the base plate. The generated fundamental SH waves propagate in the radial direction of the base plate. Defects scatter the waves, and reflections are received by the transducer. A synthetic aperture focusing technique based on the so-called exploding reflector model is applied to image the tank bottom. Wu et al. [11] then demonstrate a $12 \mathrm{~dB}$ improvement in the amplitude of SH waves generated by a magnetostrictive transducer when a soft magnetic patch is applied, which reduces the resistance of the magnetic circuit. This optimization of the magnetic circuit is also applicable to transducers that send and receive torsional waves in pipes. Crespo et al. [12] propose an experimental method to construct the Lamb wave and $\mathrm{SH}$ wave dispersion curves for a plate using a transmitter and two receivers, which requires no knowledge of the material properties. The paper by Rucka et al. [13] completes the applications to plates by investigating the integrity of the adhesive in single-lap joints between aluminum plates by images processed from the wavefield acquired by scanning laser Doppler vibrometer and the weighted root mean square.

Axial transmission of ultrasound in human cortical bone is under development as a biomarker for early detection of osteoporosis and fracture risk. While the geometry of human bones, such as the tibia, is far from the geometry of plates, it is common to use Lamb waves as surrogates for waves in cortical bones. Okumura et al. [14] developed a rapid high-resolution technique to determine the wavenumbers of propagating waves in surrogates for human bone.

One manuscript addresses Rayleigh waves propagating along the surface of a half-space. Wang et al. [15] provide forward and inverse analyses of Rayleigh wave scattering from surface breaking defects based on reflection coefficients in order to predict the shape of realistic surface-breaking flaws.

The final four manuscripts investigate defect localization for long-range inspection of different types of one-dimensional waveguides. Zhang et al. [16] considered long-range ultrasonic guided waves in a steel square bar and analyzed the non-detection zone for flaws located near the back wall. Xing et al. [17] propose a single mode extraction algorithm to precisely localize defects in rail. Zhang et al. [18] analyze guided waves, including second harmonic generation associated with the contact acoustic nonlinearity, in multi-strand cable where transducers are coupled to only two strands in the cable. Finally, Chang and Moon [19] investigate accurate group velocity of electromagnetic waves in dispersive waveguides, such as insulated cables, in which defect localization is performed using reflectometry.

Funding: This research received no external funding.

Acknowledgments: I would like to thank all of the authors who submitted manuscripts to this special issue, as well as the reviewers, who helped to improve the quality of the manuscripts.

Conflicts of Interest: The author declares no conflicts of interest.

\section{References}

1. Hakoda, C.; Lissenden, C.; Shokouhi, P. Clamping resonators for low-frequency S0 lamb wave reflection. Appl. Sci. 2019, 9, 257. [CrossRef]

2. Rose, J.; Philtron, J.; Liu, G.; Zhu, Y.; Han, M. A hybrid ultrasonic guided wave-fiber optic system for flaw detection in pipe. Appl. Sci. 2018, 8, 727. [CrossRef]

3. Pedram, S.; Mudge, P.; Gan, T. Enhancement of ultrasonic guided wave signals using a split-spectrum processing method. Appl. Sci. 2018, 8, 1815. [CrossRef] 
4. Mahal, H.; Yang, K.; Nandi, A. Detection of defects using spatial variances of guided-wave modes in testing of pipes. Appl. Sci. 2018, 8, 2378. [CrossRef]

5. Nakhli Mahal, H.; Yang, K.; Nandi, A. Improved defect detection using adaptive leaky NLMS filter in guided-wave testing of pipelines. Appl. Sci. 2019, 9, 294. [CrossRef]

6. Nakhli Mahal, H.; Yang, K.; Nandi, A. Defect detection using power spectrum of torsional waves in guided-wave inspection of pipelines. Appl. Sci. 2019, 9, 1449. [CrossRef]

7. Wu, Q.; Lee, C. A modified leakage localization method using multilayer perceptron neural networks in a pressurized gas pipe. Appl. Sci. 2019, 9, 1954. [CrossRef]

8. Hakoda, C.; Lissenden, C. Using the partial wave method for wave structure calculation and the conceptual interpretation of elastodynamic guided waves. Appl. Sci. 2018, 8, 966. [CrossRef]

9. Fan, G.; Zhang, H.; Zhang, H.; Zhu, W.; Chai, X. Lamb wave local wavenumber approach for characterizing flat bottom defects in an isotropic thin plate. Appl. Sci. 2018, 8, 1600. [CrossRef]

10. Wu, J.; Tang, Z.; Yang, K.; Wu, S.; Lv, F. Ultrasonic guided wave-based circumferential scanning of plates using a synthetic aperture focusing technique. Appl. Sci. 2018, 8, 1315. [CrossRef]

11. Wu, J.; Tang, Z.; Yang, K.; Lv, F. Signal strength enhancement of magnetostrictive patch transducers for guided wave inspection by magnetic circuit optimization. Appl. Sci. 2019, 9, 1477. [CrossRef]

12. Hernandez Crespo, B.; Courtney, C.; Engineer, B. Calculation of guided wave dispersion characteristics using a three-transducer measurement system. Appl. Sci. 2018, 8, 1253. [CrossRef]

13. Rucka, M.; Wojtczak, E.; Lachowicz, J. Damage imaging in lamb wave-based inspection of adhesive joints. Appl. Sci. 2018, 8, 522. [CrossRef]

14. Okumura, S.; Nguyen, V.; Taki, H.; Haïat, G.; Naili, S.; Sato, T. Rapid high-resolution wavenumber extraction from ultrasonic guided waves using adaptive array signal processing. Appl. Sci. 2018, 8, 652. [CrossRef]

15. Wang, B.; Da, Y.; Qian, Z. Forward and inverse studies on scattering of rayleigh wave at surface flaws. Appl. Sci. 2018, 8, 427. [CrossRef]

16. Zhang, L.; Yang, Y.; Wei, X.; Yao, W. The study of non-detection zones in conventional long-distance ultrasonic guided wave inspection on square steel bars. Appl. Sci. 2018, 8, 129. [CrossRef]

17. Xing, B.; Yu, Z.; Xu, X.; Zhu, L.; Shi, H. Research on a rail defect location method based on a single mode extraction algorithm. Appl. Sci. 2019, 9, 1107. [CrossRef]

18. Zhang, P.; Tang, Z.; Lv, F.; Yang, K. Numerical and experimental investigation of guided wave propagation in a multi-wire cable. Appl. Sci. 2019, 9, 1028. [CrossRef]

19. Chang, S.; Moon, S. Compensation for group velocity of polychromatic wave measurement in dispersive medium. Appl. Sci. 2017, 7, 1306. [CrossRef] 